\title{
Protective effect of miconazole on rat myelin sheaths following premature infant cerebral white matter injury
}

\author{
XUEWEN SU ${ }^{1-3^{*}}$, WENYAN TANG ${ }^{1,2^{*}}$, ZUO LUAN $^{1,2}$, YINXIANG YANG $^{2}$, ZHAOYAN WANG $^{2}$, \\ $\mathrm{YU} \mathrm{ZHANG}^{2}$, QIAN WANG ${ }^{2}$, LEI SUO $^{1}$, ZHEN HUANG $^{1}$, XIUE WANG $^{2}$ and HAIFENG YUAN ${ }^{3}$ \\ ${ }^{1}$ Department of Pediatrics, The Third Clinical College of Southern Medical University, Guangzhou, \\ Guangdong 510515; ${ }^{2}$ Department of Pediatrics, Navy General Hospital of People's Liberation Army, Beijing 100048; \\ ${ }^{3}$ Department of Pediatrics, Inner Mongolia People's Hospital, Hohhot, Inner Mongolia 010010, P.R. China
}

Received January 3, 2017; Accepted September 13, 2017

DOI: $10.3892 / e t m .2018 .5717$

\begin{abstract}
The aim of the present study was to investigate the protective effects of miconazole on myelin sheaths following cerebral white matter damage (WMD) in premature infant rats. Sprague Dawley rats (3-days-old) were randomly divided into four groups $(\mathrm{n}=30$ each) as follows: Sham surgery group, WMD model group, $10 \mathrm{mg} / \mathrm{kg} /$ day treatment group and $40 \mathrm{mg} / \mathrm{kg} / \mathrm{day}$ treatment group. A cerebral white matter lesion model was created by ligating the right common carotid artery for $80 \mathrm{~min}$. Treatment groups were administered with 10 or $40 \mathrm{mg} / \mathrm{kg}$ miconazole at 4-8 days following birth (early treatment group) or 5-11 days following birth (late treatment group). Rats in the model group received the same concentration of dimethylsulfoxide. Myelin basic protein (MBP) immunohistochemical staining and western blotting were used to detect the expression of cerebral white matter-specific MBP, and changes in myelin structure were observed using transmission electron microscopy. No swelling or necrosis was observed in the corpus callosum of the sham group rats, whereas rats in the model group demonstrated edema, loose structure, fiber disorder, inflammatory gliocytes and selective white matter lesions. Following treatment with miconazole, MBP expression in the corpus callosum was significantly higher compared with the model group. Furthermore, in the model group, myelin sheaths in the corpus callosum were loose with small vacuoles, there was a marked decrease in thickness and structural damage was observed. Conversely, a marked improvement in myelination was observed in the treatment group. The results of the present study suggest that
\end{abstract}

Correspondence to: Dr Zuo Luan, Department of Pediatrics, Navy General Hospital of People's Liberation Army, 6 Fucheng Road, Beijing 100048, P.R. China

E-mail: zhibingpi@sina.com

${ }^{*}$ Contributed equally

Key words: premature infant cerebral white matter lesions, miconazole, myelin basic protein miconazole is able to promote formation of the myelin sheath to ameliorate premature cerebral white matter lesions caused by ischemia or hypoxia in rats.

\section{Introduction}

Oligodendrocytes (OLs) are major cells of the central nervous system (CNS) and are critical for neuronal function (1). CNS myelin abnormalities are features of several neurological disorders (2), including white matter injury (3), multiple sclerosis (4) and neuromyelitis optica (5). The causes of demyelination typically include immunological injury, excitotoxicity, viral infection, dystrophia and oxidative stress (6). At present, there are two methods used to treat demyelinating diseases; the first is reducing demyelination and the second is promoting remyelination (7). The therapeutic approaches include transplanting OL progenitor cells (OPCs) to protect endogenous OLs, or increasing the activity of exogenous OPCs to reduce demyelination and promote remyelination (8). Several studies have reported that the survival rate of transplanted OPCs is low, that few OPCs differentiate into mature OLs and that transplanting exogenous OPCs can lead to host rejection (9). Therefore, the aim of the present study was to explore a more effective method of promoting myelination by protecting and increasing the activity of endogenous OPCs.

Stimulating an endogenous regenerative response may be a potential effective treatment for chronic demyelinating diseases, including multiple sclerosis. Recently, Najm et al (10) reported that miconazole promotes OPC differentiation and induces remyelination in demyelinating disease models via mitogen-activated protein kinase (MEK)-dependent activation of extracellular signal-regulated kinase (ERK)1/2. Their study investigated the miconazole-promoted remyelination in a lysolecithin-induced demyelinating adult mouse model. In the present study, whether miconazole also serves a therapeutic role in premature infant cerebral white matter lesions was investigated.

White matter damage (WMD) is the most common type of cerebral lesion and cause of neural-developmental disorders, including premature cognitive defects and cerebral palsy, in premature infants (11). The pathogenesis of WMD typically includes changes in cerebral blood flow and cerebral tissue metabolism, which leads to a loss of OPCs and mature OLs, 
causing cerebral white matter lesions (12). Ischemic hypoxia and infection are the main causes of cerebral white matter lesions in premature infants (13). At present, there is no effective treatment for premature infants with hypoxia and ischemic cerebral lesions. The aim of the present study was to explore the effect of miconazole on myelin regeneration and formation in the corpus callosum of rats with WMD.

\section{Materials and methods}

Experimental animals. Sprague-Dawley dams with litter were purchased from Beijing Vital River Laboratory Animal Technology Co., Ltd. (Beijing, China). A total of 120 3-day-old Sprague Dawley rat pups (62 males and 58 females, mean weight, 7-8 g) from 12 litters were housed in a clean environment at $15-28^{\circ} \mathrm{C}$ with $45-44 \%$ humidity and a $12 \mathrm{~h}$ light/dark cycle, and provided with adequate food and water. All experimental procedures were approved by the Animal Ethical and Welfare Committee of the Navy General Hospital of People's Liberation Army (Beijing, China).

Grouping and drug delivery. Experimental animals were randomly divided into four groups $(n=30 /$ group): The sham surgery, model, $10 \mathrm{mg} / \mathrm{kg}$ miconazole treatment and $40 \mathrm{mg} / \mathrm{kg}$ miconazole treatment groups. The treatment groups were administered with intraperitoneal injections of 10 or $40 \mathrm{mg} / \mathrm{kg} /$ day miconazole (Sigma-Aldrich; Merck KGaA; Darmstadt, Germany) at 4-8 days of age (the early treatment group consisting of 10 rats from each group) or 5-11 days of age (the late treatment group consisting of 10 rats from each group). The model group was administered with intraperitoneal injections of dimethylsulfoxide (DMSO) at the same concentration, whereas rats in the sham group received no treatment.

Establishment of the WMD model. At 3 days old, the rats were anesthetized using sodium pentobarbital (Shanghai Xinya Pharmaceutical, Co., Ltd., Shanghai, China in a sterile environment. The limbs were fixed in the supine position on the operating table, the skin was sterilized with iodine, and a midline incision was made at the neck to reveal the right common carotid artery, internal jugular vein and vagus nerve. The right common carotid artery was ligated and the skin incision was subsequently sutured (14). In the sham group, only the right common carotid artery was exposed. Rats were placed on a heating mat at $37^{\circ} \mathrm{C}$ following the surgery for recovery, and subsequently housed in an environment containing $6 \%$ oxygen and $94 \%$ nitrogen at $33^{\circ} \mathrm{C}$ to provide constant anoxia for $80 \mathrm{~min}$ before being returned to their mother's cage.

Brain tissue sections. At either days 8 or days 12 post-surgery, rats ( $n=3$ per group) were anesthetized by intraperitoneal injection with pentobarbital sodium $(50 \mathrm{mg} / \mathrm{kg})$, the thoracic cavity was opened and the right auricle was dissected. A total of $\sim 100 \mathrm{ml}$ saline followed by $\sim 100 \mathrm{ml}$ paraformaldehyde were perfused into vascular system via the left ventricle. The rats were then decapitated, and brain tissue was harvested and fixed in $4 \%$ paraformaldehyde for $24 \mathrm{~h}$ at $4^{\circ} \mathrm{C}$. Tissues were subsequently dehydrated in $30 \%$ sucrose solution until the tissue blocks sank to the bottom. Serial sections of the desiccated brain tissue (10- $\mu$ m-thick) were then cut.
Hematoxylin and eosin $(H \& E)$ staining. Brain tissue were fixed in $4 \%$ paraformaldehyde solution at room temperature for $24 \mathrm{~h}$, embedded in paraffin and cut into continuous sections (5- $\mu \mathrm{m}$-thick) starting from the anterior fontanel. Sections were dehydrated in a graded series of alcohol, washed in distilled water and stained with hematoxylin for $3 \mathrm{~min}$ at room temperature. Sections were submerged in $1 \%$ hydrochloric acid for $15 \mathrm{sec}$, washed and immersed in $0.5 \%$ eosin (alcoholic) solution (cat no. G1001; Wuhan Servicebio Biotechnology Co., Ltd., Wuhan, China) for 3-5 min at room temperature. Sections were subsequently mounted and observed under an optical microscope at a magnification of x 200 .

Myelin basic protein (MBP) immunohistochemical staining. Brain tissue sections were washed in PBS, digested in $0.03 \%$ Triton X-100 and blocked with 5\% sheep serum (Gibco; Thermo Fisher Scientific, Inc., Waltham, MA, USA) for $1 \mathrm{~h}$ at $18-24^{\circ} \mathrm{C}$. Tissues were incubated in anti-MBP antibodies (cat no. ab62631; Abcam, Cambridge, UK; 1:200) at $4^{\circ} \mathrm{C}$ overnight, washed three times with PBS and then incubated with goat anti mouse secondary antibodies (1:500; cat no. 115-165-068; Jackson ImmunoResearch Europe, Ltd., Newmarket, UK) for $2 \mathrm{~h}$ at room temperature in the dark. The sections were washed again three times with PBS, observed under a fluorescence microscope and images were captured at a magnification of x200. ImagePro Plus 6.0 software (Media Cybernetics, Inc., Rockville, MD, USA) was used to convert the fluorescent images into black and white images, and the integrated optical density (IOD) value was measured.

Western blotting. At either days 8 or days 12 post-surgery, protein was extracted from the corpus callosum of rats $(n=3$ per group) using a Minute ${ }^{\mathrm{TM}}$ Total Protein Extraction kit (cat no. SD-001/SN-002; Invent Biotechnologies, Co., Ltd, Beijing, China) according to the manufacturer's protocols and quantified as previously described (10). Samples were denatured and equal amounts of protein (30 $\mu \mathrm{g} /$ lane) were loaded, separated by $10 \%$ SDS-PAGE and transferred to a PVDF membrane overnight. The membrane was blocked with $5 \%$ skim milk for $30 \mathrm{~min}$ at room temperature, incubated with anti-MBP antibodies $(1: 1,000)$ for $12 \mathrm{~h}$ at $4^{\circ} \mathrm{C}$ and subsequently incubated with horseradish peroxidase-conjugated goat anti mouse secondary antibodies for $30 \mathrm{~min}$ at room temperature. Protein bands were developed using enhanced chemiluminescence (cat no. 29050; Engreen Co., Ltd, Beijing, China). ImageJ software 2.1.4.7 (National Institutes of Health, Bethesda, MA, USA) was used to calculate the expression of MBP relative to GAPDH (cat no. GB12002; Wuhan Servicebio Biotechnology Co., Ltd, Wuhan, China) via densitometry. The dilution of the anti-GAPDH antibody was 1:1,000.

Transmission electron microscopy. At either days 8 or days 12 post-surgery, sections (1-3 mm thick) from the right corpus callosum of rats ( $n=3$ per group) were fixed in $2 \%$ glutaraldehyde at $4^{\circ} \mathrm{C}$ for $72 \mathrm{~h}$ and then in $1 \%$ osmic acid at $4^{\circ} \mathrm{C}$ for $2 \mathrm{~h}$. The sections were dehydrated using a series of $30-100 \%$ acetone and embedded in epoxy resin embedding medium. Myelin cross-sectional slices (50-nm-thick) were obtained using an ultramicrotome. Sections were observed under a transmission electron microscope and images were 
captured for further analysis. A total of 800 root axonal fibers were counted at a magnification of x15,000. Following image acquisition, axon and myelin diameters were measured using Image-Pro Plus 6.0 software (Media Cybernetics, Inc.) and the G-ratio value (axon diameter/myelin diameter) was calculated as previously described (15).

Neuroethology tests. The following neuroethology tests were performed in a blinded manner at postnatal day 28 .

Tail suspension test. A tail suspension test was performed as previously reported (16), with minor modifications. Rat forelimbs were allowed to grasp a glass rod (diameter, $0.5 \mathrm{~cm}$ ) at a height of $45 \mathrm{~cm}$, and the time taken between being placed on the rod and falling off was recorded and scored. Scoring was as follows: $<10 \mathrm{sec}, 1$ point; $>10-30 \mathrm{sec}, 2$ points; $>30-120 \mathrm{sec}$, 3 points; $>120-300 \mathrm{sec}, 4$ points; $>300 \mathrm{sec}, 5$ points.

Slope test. Rats were placed with their heads at a $45^{\circ}$ decline and the time taken for rats to turn their heads up $>135^{\circ}$ was recorded (17).

Field test. The bottom of a $30 \times 30 \times 30 \mathrm{~cm}$ square box was divided into nine equal squares with chalk lines. Rats were placed in the central square and their activity was observed for $30 \mathrm{~min}$. Scoring was performed as follows: 1 point for each instance where $>50 \%$ of the rat's body moved to an adjacent square and 1 point whenever the rat was observed standing on hind legs.

Statistical analysis. Data are presented as the mean \pm standard deviation and statistical analysis was performed using SPSS 21.0 software (IBM Corp., Armonk, NY, USA). One-way ANOVA followed by a post-hoc Tukey's test was used to examine differences between multiple groups. $\mathrm{P}<0.05$ was considered to indicate a statistically significant difference.

\section{Results}

Successful establishment of the WMD model. At 4 days post-surgery, H\&E staining revealed that the corpus callosum structure in rats from the sham group was normal; cells had a dense and uniform distribution, the brain tissue outline was clear, and no cell swelling, necrosis or pathological changes were observed (Fig. 1A and B). Conversely, swelling, necrosis, nucleus pycnosis, loose structure, fiber disorder, enlarged lateral ventricles, hyperplastic inflammatory gliocytes, and active and selective white matter lesions were observed in the model group (Fig. 1C and D). MBP immunohistochemical staining results demonstrated that the corpus callosum in the sham group had no distinct myelin loss (Fig. 2A and B); however, significant myelin loss was observed in the white matter outside the sac on the surgical side of the model group compared with the non-surgical side and the sham group $(\mathrm{P}<0.05$; Fig. 2C-E). These results indicate that the WMD model was successfully established. callosum of rats with WMD. MBP immunohistochemical

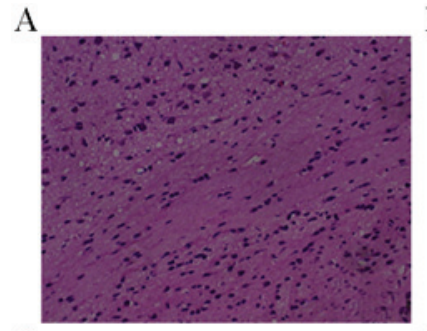

$\mathrm{B}$
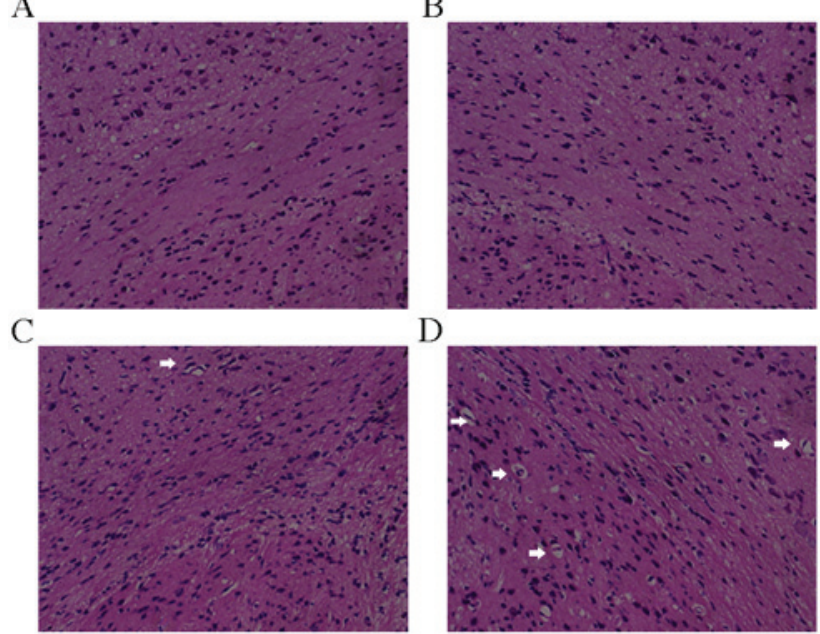

$\mathrm{D}$

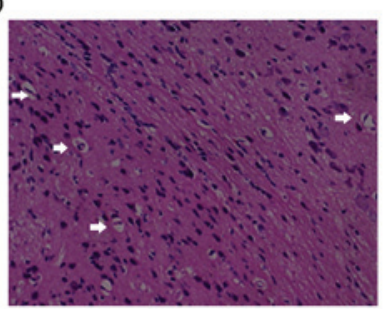

Figure 1. Hematoxylin and eosin staining of the corpus callosum in the (A and B) sham and (C and D) model groups. (C) In the model group, cell swelling was observed on the left non-surgical side, however there were no necrotic changes. (D) Cerebral white matter cells in the surgical right side of the model group exhibited swelling, necrosis, nuclear pycnosis, a loose structure, fiber disorder, enlarged lateral ventricles, inflammatory gliocytes and selective white matter lesions. The indicate swelling necrosis cells and demyelination. Magnification, x200.
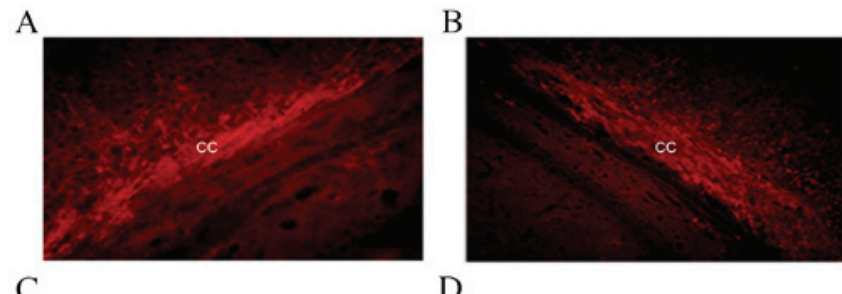

$\mathrm{C}$
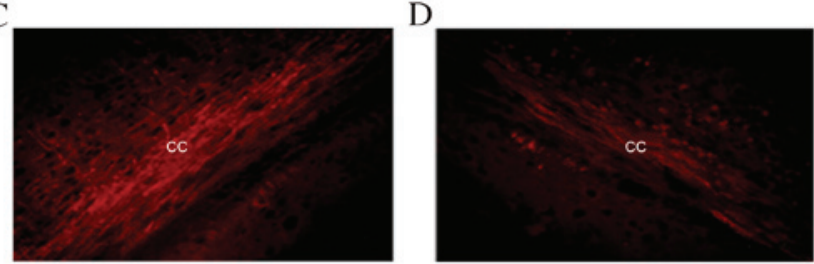

E

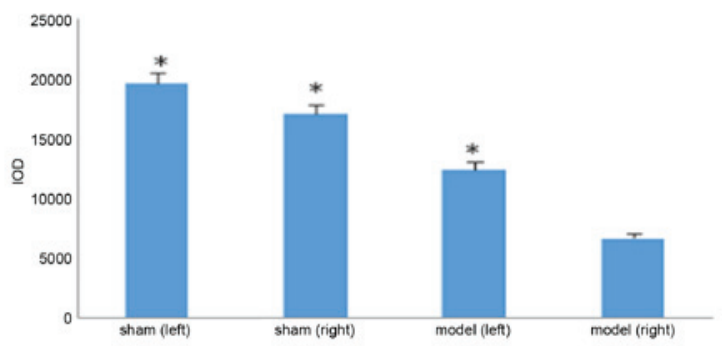

Figure 2. MBP immunohistochemical staining of the $\mathrm{CC}$ in the (A) left and (B) right (surgical) side in the sham group. No loss of myelin was observed on either side. MBP immunohistochemical staining of the $\mathrm{CC}$ in the $(\mathrm{C})$ left and (D) right (surgical) side in the model group. No significant myelin loss was observed on the left side, whereas myelin loss was evident on the surgical side. (E) Quantification of MBP by IOD. Magnification, $\mathrm{x} 200$. $^{*} \mathrm{P}<0.05$ vs. the model group right side. MBP, myelin basic protein; $\mathrm{CC}$, corpus callosum; IOD, integrated optical density.

staining results revealed significant myelin loss on the surgical side of the corpus callosum in the model control group $(\mathrm{P}<0.05$; Fig. 2E). Rats treated with 10 or $40 \mathrm{mg} / \mathrm{kg} /$ day miconazole in 

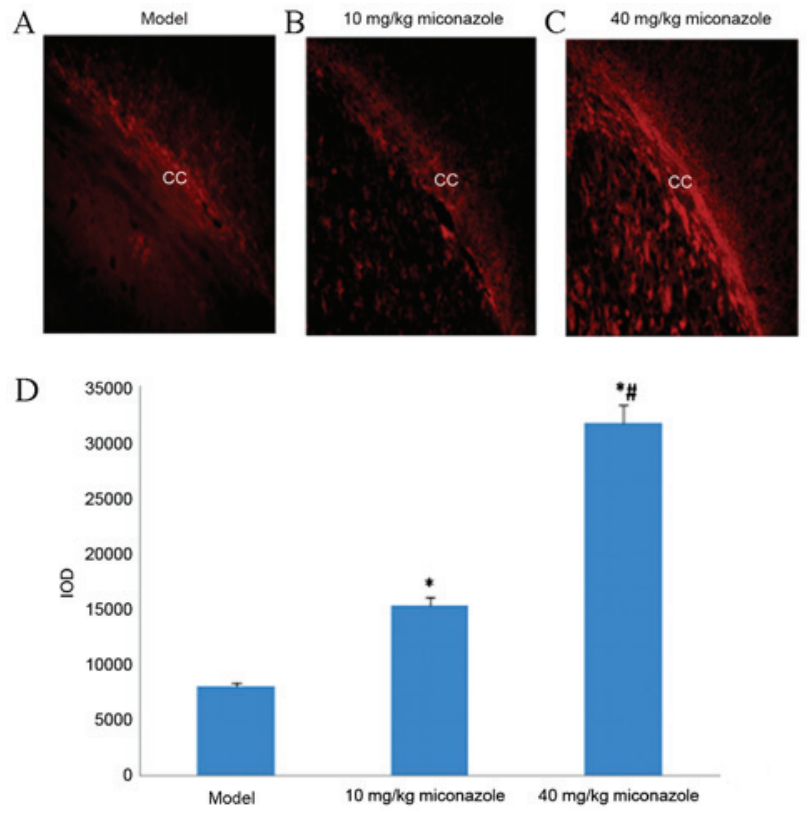

Figure 3. Early treatment with miconazole enhances myelination in vivo. Representative immunohistochemical images of the lateral CC of (A) model, (B) $10 \mathrm{mg} / \mathrm{kg} / \mathrm{day}$ miconazole treatment and (C) $40 \mathrm{mg} / \mathrm{kg} /$ day miconazole treatment rats in the early treatment group. MBP staining (red) revealed the extent of developmental myelination. (D) Quantification of MBP by IOD. Magnification, $x 200 .{ }^{*} \mathrm{P}<0.05$ vs. the model group; ${ }^{\prime} \mathrm{P}<0.05$ vs. the $10 \mathrm{mg} / \mathrm{kg} /$ day miconazole treatment group. CC, corpus callosum; MBP, myelin basic protein; IOD, integrated optical density.
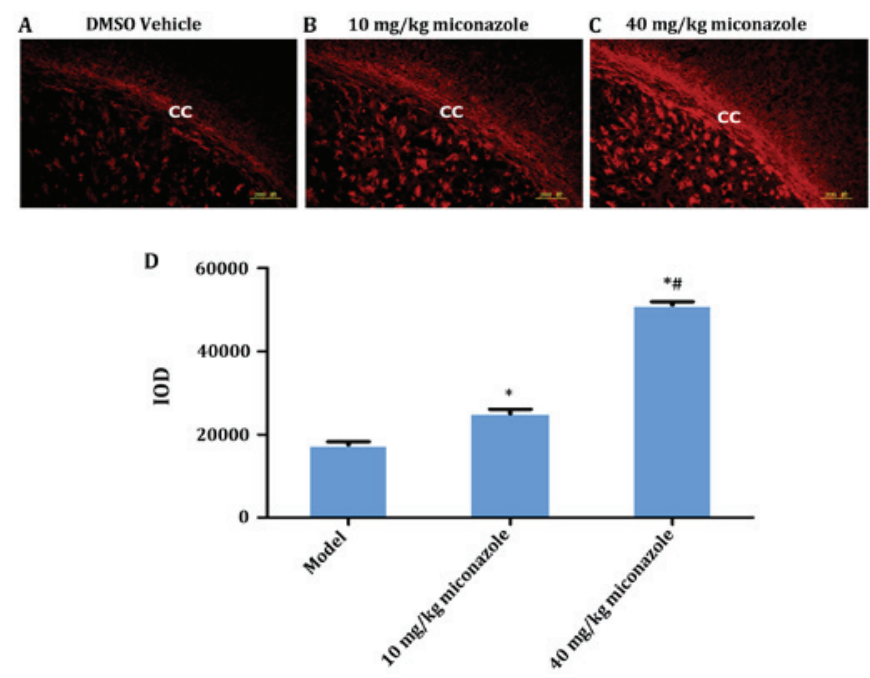

Figure 4. Late treatment with miconazole enhances myelination in vivo. Representative immunohistochemical images of the lateral CC of (A) model, (B) $10 \mathrm{mg} / \mathrm{kg} /$ day miconazole treatment and (C) $40 \mathrm{mg} / \mathrm{kg} /$ day miconazole treatment rats in the late treatment group. MBP staining (red) revealed the extent of developmental myelination. (D) Quantification of MBP by IOD. Magnification, $\mathrm{x} 200{ }^{*} \mathrm{P}<0.05$ vs. the model group; ${ }^{\#} \mathrm{P}<0.05$ vs. the $10 \mathrm{mg} / \mathrm{kg} /$ day miconazole treatment group. CC, corpus callosum; MBP, myelin basic protein; IOD, integrated optical density.

the early (Fig. 3) and late (Fig. 4) treatment groups demonstrated a significantly higher expression of MBP compared with the model group $(\mathrm{P}<0.05)$. Furthermore, the IOD of rats in the $40 \mathrm{mg} / \mathrm{kg} /$ day treatment group was significantly higher compared with the rats treated with $10 \mathrm{mg} / \mathrm{kg} / \mathrm{day}$ miconazole in the early and late treatment groups $(\mathrm{P}<0.05)$.

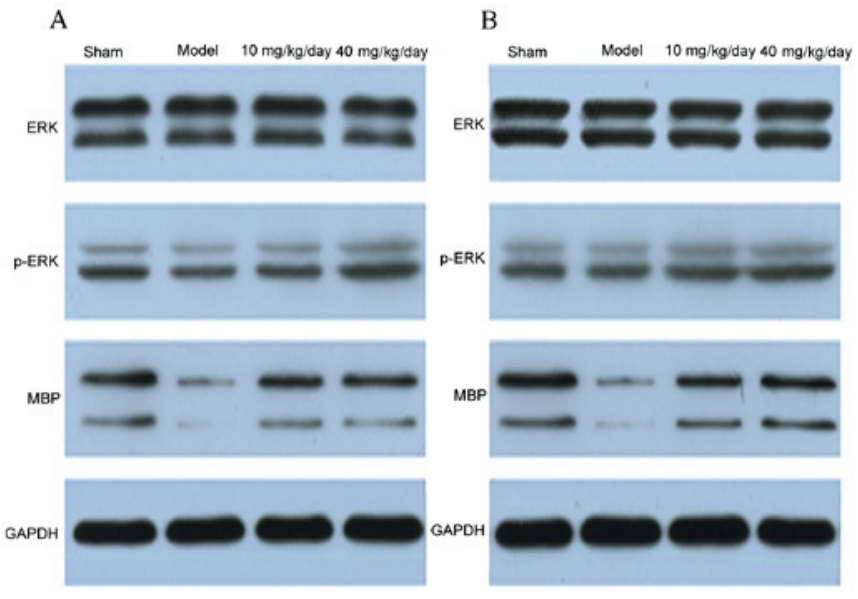

Figure 5. Western blotting of MBP, p-ERK and ERK protein levels in the (A) early and (B) late treatment groups. GAPDH was used as an internal control. MBP, myelin basic protein; p, phosphorylated; ERK, extracellular-signal regulated kinase.
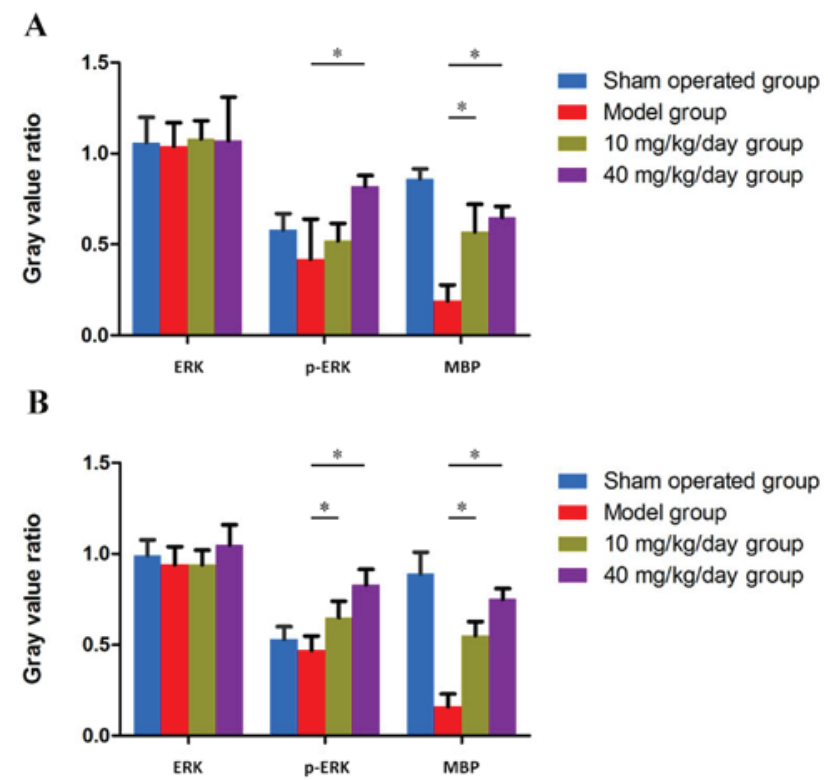

Figure 6. Quantification of MBP, ERK and p-ERK in the (A) early and (B) late treatment groups. ${ }^{*} \mathrm{P}<0.05$ vs. sham group. $\mathrm{MBP}$, myelin basic protein; $\mathrm{p}$, phosphorylated; ERK, extracellular-signal regulated kinase.

Miconazole treatment increases MBP expression and enhances ERK1/2 activity in the corpus callosum of rats with $W M D$. Western blotting results also revealed that the protein expression of MBP was increased in the miconazole treatment groups compared with the model group $(\mathrm{P}<0.05)$; however, no significant differences were observed between the 40 and $10 \mathrm{mg} / \mathrm{kg} /$ day groups (Figs. 5 and 6). These results indicate that miconazole is able to alter the protein expression of MBP in the corpus callosum, which suggests that it exerts a protective role during hypoxic ischemia. Furthermore, miconazole significantly upregulated MBP expression and enhanced ERK1/2 activity, as revealed by increased phosphorylated-ERK expression compared with the model group.

Miconazole treatment improves the morphological features of WMD. Transmission electron microscopy results revealed 
Table I. Neuroethology test results.

\begin{tabular}{lccc}
\hline Group & $\begin{array}{c}\text { Suspension } \\
\text { test }\end{array}$ & $\begin{array}{c}\text { Slope } \\
\text { test }(\mathrm{sec})\end{array}$ & Field test \\
\hline Sham operation & $3.6 \pm 0.97^{\mathrm{a}-\mathrm{c}}$ & $3.2 \pm 0.92^{\mathrm{a}-\mathrm{c}}$ & $13.4 \pm 1.43^{\mathrm{a}-\mathrm{c}}$ \\
Model & $1.5 \pm 0.53^{\mathrm{b}, \mathrm{c}}$ & $9.6 \pm 1.5^{\mathrm{b}, \mathrm{c}}$ & $5.9 \pm 1.73^{\mathrm{b}, \mathrm{c}}$ \\
$10 \mathrm{mg} / \mathrm{kg}$ miconazole & $2.4 \pm 0.84^{\mathrm{c}}$ & $6.6 \pm 1.1^{\mathrm{c}}$ & $7.3 \pm 1.34^{\mathrm{c}}$ \\
$40 \mathrm{mg} / \mathrm{kg}$ miconazole & $2.7 \pm 0.67$ & $6.9 \pm 1.6$ & $7.5 \pm 1.35$ \\
P-value & $<0.05^{\mathrm{d}}$ & $<0.05^{\mathrm{e}}$ & $<0.05^{\mathrm{f}}$ \\
\hline
\end{tabular}

${ }^{\mathrm{a}} \mathrm{P}<0.05$ vs. model group, ${ }^{\mathrm{b}} \mathrm{P}<0.05$ vs. $10 \mathrm{mg} / \mathrm{kg}$ group, ${ }^{\mathrm{c}} \mathrm{P}<0.05$ vs. $40 \mathrm{mg} / \mathrm{kg}$ group, ${ }^{\mathrm{d}} \mathrm{F}$ value $=12.617$, ${ }^{\mathrm{e}} \mathrm{F}$ value $=40.419$, and ${ }^{\mathrm{f}} \mathrm{F}$ value $=51.154$.
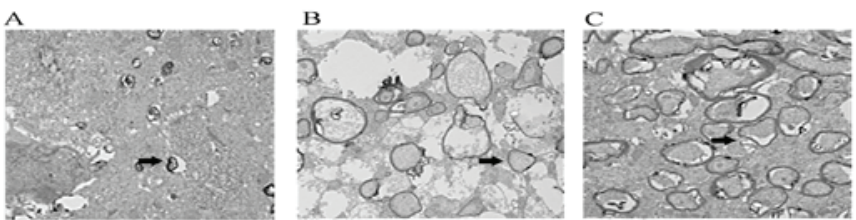

D
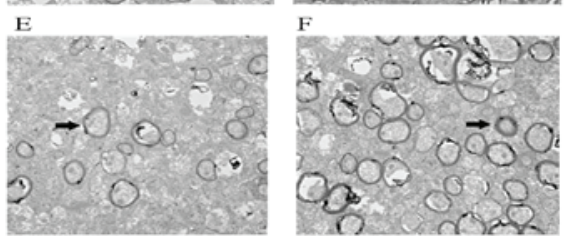

Figure 7. Electron micrographs showed the protective effect of miconazole on rat myelin sheaths. (A) control, (B) $10 \mathrm{mg} / \mathrm{kg} / \mathrm{day}$ miconazole treatment group and (C) $40 \mathrm{mg} / \mathrm{kg} /$ day miconazole treatment group were the early treatment groups. The (D) control, (E) $10 \mathrm{mg} / \mathrm{kg} / \mathrm{day}$ miconazole treatment group and (F) $40 \mathrm{mg} / \mathrm{kg} /$ day miconazole treatment group were the late treatment groups. The protective effect of $40 \mathrm{mg} / \mathrm{kg} /$ day treatment ( $\mathrm{C}$ and $\mathrm{F}$ ) was better than that of $10 \mathrm{mg} / \mathrm{kg} / \mathrm{day}$ (B and E) treatment. Arrows indicate the improvement of demyelination. Magnification, x15,000.

that, at either day 8 or day 12 post-surgery, little myelination was present in the model group, nerve fibers were irregularly shaped and arranged loosely, and myelin thickness was markedly decreased (Fig. 7). Following miconazole treatment, the quantity of myelin was markedly increased compared with the control group, indicating that demyelination was ameliorated and myelin thickness was improved. G-ratio analysis demonstrated that the G-ratio in the control group was significantly higher compared with the miconazole treatment groups, while the $40 \mathrm{mg} / \mathrm{kg}$ miconazole treatment group G-ratio value was lower compared with the $10 \mathrm{mg} / \mathrm{kg}$ treatment group (data not shown). These results suggest that miconazole is able to improve cerebral white matter injury in premature infants.

Miconazole treatment improves the neurological function of rats with WMD. Neuroethology tests were performed to assess the effect of miconazole treatment on the functioning of rats with WMD (Table I). In the tail suspension test, rats in the miconazole treatment groups demonstrated flexibility, agility and accuracy of movement, and were able to turn and crawl along the glass rod with a long suspension time. Compared with the treatment groups, rats in the model group were unresponsive with decreased autokinetic movement, flexibility and stability. Furthermore, they exhibited shaky lower limbs and were unable to stay on the rod for extended periods. Rats in the sham group scored significantly higher in the suspension test compared with the model and treatment groups $(\mathrm{P}<0.05)$. In the slope test, rats in the sham group took a significantly shorter amount of time to move compared with the model and treatment groups $(\mathrm{P}<0.05)$; however, the times recorded for the treatment groups were significantly shorter compared with the model group $(\mathrm{P}<0.05)$. Lastly, in the field test the sham group scored significantly higher compared with the model and treatment groups $(\mathrm{P}<0.05)$, whereas the model group scored significantly lower compared with the treatment groups $(\mathrm{P}<0.05)$ (Table I). No significant differences were observed between the 10 and $40 \mathrm{mg} / \mathrm{kg} / \mathrm{day}$ miconazole treatment groups for any of the neuroethology tests.

\section{Discussion}

Cerebral white matter lesions in premature infants may cause long periods of movement and cognitive dysfunction, which is one of the main causes of cerebral palsy and mental retardation in children (11). Infants with very low birth weights $(<1,500 \mathrm{~g})$ have a high risk of premature infant cerebral white matter lesions, with 10-15\% developing movement dysfunction, and $25-50 \%$ presenting with cognitive and behavioral deficits or social dysfunction (18). This is the primary cause of nervous system developmental disorders in premature infants and negatively affects survival (18). At present, there is no effective treatment for WMD (19). Cerebral OPC injury is the main cause of WMD, resulting in myelin destruction and delayed remyelination (20). In recent years, researchers have performed exogenous OPC transplants in animal models in order to verify whether the OPCs are able to survive, differentiate into mature OLs and promote myelination (21). Webber et al (21) transplanted OPCs into portal vein ligation model rats and reported that, 7 weeks later, the majority of transplanted cells had survived and expressed OPC-specific markers, including neural/glial antigen 2, O-antigen 4 and OL-transcription factor 2 , indicating that the transplanted OPCs did not differentiate into mature OLs. Another study transplanted OPCs induced from human neural stem cells into rat demyelination models; at 8 weeks the majority of cells were alive, but $<2 \%$ expressed MBP, a specific marker of mature OLs (9). These results indicate that, although transplanted OPCs are able to migrate to the injury and survive for an extended period, the majority do not differentiate into mature OLs. This is similar to previous observations of WMD; when demyelinating lesions occur, endogenous OPCs migrate to the lesion site, but few differentiate into mature OLs (22). It has been reported that the microenvironment of the CNS is an important factor for neuron and axonal regeneration (23); as such, efforts have been made to improve the microenvironment when attempting to identify effective targets for the promotion of axonal regeneration (24).

The aim of the present study was to promote endogenous OPC survival, differentiation and myelin formation in order to treat WMD. Muscarine receptor antagonists, including benztropine and clemastine, have previously been reported to improve multiple sclerosis and promote myelin regeneration (10). The results of the present study demonstrated that miconazole, 
another member of the family that has been approved by the US Food and Drug Administration as a fungal infection treatment (10), is also able to promote OPC differentiation in vitro. Miconazole is also able to promote lysolecithin-induced demyelinating rat endogenous OPC differentiation, where the number of mature OLs is $13 x$ that of the controls and axon myelination is almost $12 \mathrm{x}(10)$. This indicates that miconazole serves an important regulatory role in the early stages of OPC differentiation. In the present study, it was investigated whether miconazole was also able to promote endogenous OPC differentiation in a rat model of infant WMD and whether it induced myelin formation following ischemia or hypoxia.

The CNS of 7-day-old rats is the same as that of term or near-term neonates, which has formed early myelin (25). Unilateral carotid artery ligation combined with hypoxia induces brain lesions in the unilateral cortex and hippocampal neurons, whereas it does not produce significant white matter lesions. Rat cerebral white matter is vulnerable 2-3 days after birth, at which point it consists mainly of OPCs (26). As such, immature 3-day-old rats are ideal for studying premature cerebral white matter injury (26). In the present study, H\&E staining and MBP immunohistochemical staining was used to verify that the WMD model was successfully established following carotid artery ligation. H\&E staining revealed swollen cells, dilated intercellular spaces, a disordered cellular arrangement, tiny infarcts and white matter injuries of the corpus callosum in the model group, and MBP immunohistochemical staining revealed significant demyelination. These results indicate that the animal model of hypoxic ischemic cerebral white matter injury was successful.

The protective effect and underlying mechanisms of miconazole on the myelin sheath in 3-day-old rats with cerebral white matter injury were also investigated. The experimental results provide a basis for the potential clinical use of miconazole as a treatment for WMD. MBP immunohistochemical staining and western blotting results revealed that MBP expression was higher in the corpus callosum of miconazole-treated rats compared with the model controls, and that treatment with $40 \mathrm{mg} / \mathrm{kg}$ was more effective compared with $10 \mathrm{mg} / \mathrm{kg}$. The results of transmission electron microscopy further indicated that treatment with miconazole ameliorates ischemia-induced demyelination and loss of myelin sheath thickness. Miconazole may therefore be beneficial for improving myelin dysplasia in premature infants with cerebral white matter injury. In addition, the results of neurobehavioral tests demonstrated that miconazole effectively improved ischemia-induced neurobehavioral dysfunction in the immature rats.

Miconazole was also demonstrated to enhance the activity of ERK. It has been reported that the ERK signaling pathway serves a role in oligodendroglial and myelin development in the CNS (27-32). Furthermore, Xie et al (33) suggested that the suppression of proto-oncogene tyrosine-protein kinase Fyn/MEK/ERK phosphorylation inhibits the maturation of OPCs, leading to axonal hypomyelination. In the present study, it was demonstrated that miconazole significantly upregulated MBP expression and enhanced ERK1/2 activity, as revealed by the increased expression of phosphorylated ERK compared with the model group. Furthermore, the present study demonstrated that the systemic delivery of miconazole contributes to improved neurobehavioral functions. However, miconazole is currently only approved for topical administration in humans (34). Significant optimization of dosing, delivery and chemical structure is required to enhance the on-target pharmacology of miconazole in OPCs, and diminish any potential off-target side effects. The ability of miconazole to cross the blood-brain barrier raises the possibility that these agents, or modified derivatives of them, may advance into clinical trials for the treatment of WMD.

Inconclusion, the present study provides evidence that miconazole is able to significantly reduce ischemia/anoxia-induced WMD via promoting myelination in a rat model of ischemic brain injury. This protection is implemented via the activation of ERK phosphorylation. The protective effect of miconazole in the rat brain makes it an ideal candidate for further investigation as a clinical treatment for WMD.

\section{Acknowledgements}

The present study was supported by the fund of The National Key R\&D Program of China (grant no. 2017YFA0104200), which was given to Dr Zuo Luan.

\section{References}

1. Lopez Juarez A, He D and Richard Lu Q: Oligodendrocyte progenitor programming and reprogramming: Toward myelin regeneration. Brain Res 1638: 209-220, 2016.

2. Chew LJ and DeBoy CA: Pharmacological approaches to intervention in hypomyelinating and demyelinating white matter pathology. Neuropharmacology 110: 605-625, 2016.

3. Ryan M, Ibrahim M and Parmar HA: Secondary demyelination disorders and destruction of white matter. Radiol Clin North Am 52: 337-354, 2014.

4. Bando Y, Nomura T, Bochimoto H, Murakami K, Tanaka T, Watanabe $\mathrm{T}$ and Yoshida S: Abnormal morphology of myelin and axon pathology in murine models of multiple sclerosis. Neurochem Int 81: 16-27, 2015.

5. Jeong IH, Choi JY, Kim SH, Hyun JW, Joung A, Lee J and Kim HJ: Comparison of myelin water fraction values in periventricular white matter lesions between multiple sclerosis and neuromyelitis optica spectrum disorder. Mult Scler 22: 1616-1620, 2016.

6. Counsell SJ, Rutherford MA, Cowan F and Edwards AD: Magnetic resonance imaging of preterm brain injury. Arch Dis Child FetalNeonatal Ed 88: F269-F274, 2003.

7. Deverman BE and Patterson PH: Exogenous leukemia inhibitory factor stimulates oligodendrocyte progenitor cell proliferation and enhances hippocampal remyelination. J Neurosci 32: 2100-2109, 2012.

8. Grulova I, Slovinska L, Blaško J, Devaux S, Wisztorski M, Salzet M, Fournier I, Kryukov O, Cohen S and Cizkova D: Delivery of alginate scaffold releasing two trophic factors for spinal cord injury repair. Sci Rep 5: 13702, 2015.

9. Jiang S, Seng S, Avraham HK, Fu Y and Avraham S: Process elongation of oligodendrocytes is promoted by the Kelch-related protein MRP2/KLHL1. J Biol Chem 282: 12319-12329, 2007.

10. Najm FJ, Madhavan M, Zaremba A, Shick E, Karl RT, Factor DC, Miller TE, Nevin ZS, Kantor C, Sargent A, et al: Drug-based modulation of endogenous stem cells promotes functional remyelination in vivo. Nature 522: 216-220, 2015.

11. Volpe JJ: Cerebral white matter injury of the premature infant-more common than you think. Pediatrics 112: 176-180, 2003.

12. Erceg S, Ronaghi M, Oria M, Roselló MG, Aragó MA, Lopez MG, Radojevic I, Moreno-Manzano V, Rodríguez-Jiménez FJ, Bhattacharya SS, et al: Transplanted oligodendrocytes and motoneuron progenitors generated from human embryonic stem cells promote locomotor recovery after spinal cord transection. Stem Cells 28: 1541-1549, 2010.

13. Welin AK, Sedin P, Lapatto R, Sultan B, Hagberg H, Gressens P, Kjellmer I and Mallard C: Melatonin reduces inflammation and cell death in white matter in the midgestation fetal sheep following umbilical cord occlusion. Pediatr Res 61: 153-158, 2007. 
14. Yuan QC, Jiang L, Zhu LH and Yu DF: Impacts of erythropoietin on vascular endothelial growth factor receptor 2 by the extracellular signal-regulated kinase signaling pathway in a neonatal rat model of periventricular white matter damage. Zhongguo Yi Xue Ke Xue Yuan Xue Bao 38: 217-221, 2016 (In Chinese).

15. Coetzee T, Fujita N, Dupree J, Shi R, Blight A, Suzuki K, Suzuki K and Popko B: Myelination in the absence of galactocerebroside and sulfatide: Normal structure with abnormal function and regional instability. Cell 86: 209-219, 1996.

16. Mineur YS, Belzung C and Crusio WE: Effects of unpredictable chronic mild stress on anxiety and depression-like behavior in mice. Behav Brain Res 175: 43-50, 2006.

17. Breu M, Zhang J, Porambo M, Pletnikov MV, Goeral K, Kakara M, Johnston MV and Fatemi A: Diffusion tensor imaging abnormalities in the cerebral white matter correlate with sex-dependent neurobehavioral deficits in adult mice with neonatal ischemia. Dev Neurosci 38: 83-95, 2016.

18. Anderson PJ: Neuropsychological outcomes of children born very preterm. Semin Fetal Neonatal Med 19: 90-96, 2014

19. Barateiro A and Fernandes A: Temporal oligodendrocyte lineage progression: In vitro models of proliferation, differentiation and myelination. Biochim Biophys Acta 1843: 1917-1929, 2014.

20. Nistor GI, Totoiu MO, Haque N, Carpenter MK and Keirstead HS: Human embryonic stem cells differentiate into oligodendrocytes in high purity and myelinate after spinal cord transplantation. Glia 49: 385-396, 2005.

21. Webber DJ, van Blitterswijk M and Chandran S: Neuroprotective effect of oligodendrocyte precursor cell transplantation in a long-term model of periventricular leukomalacia. Am J Pathol 175: 2332-2342, 2009.

22. Chong SY and Chan JR: Tapping into the glial reservoir: Cells committed to remaining uncommitted. J Cell Biol 188: 305-312, 2010.

23. Dooley D, Vidal P and Hendrix S: Immunopharmacological intervention for successful neural stem cell therapy: New perspectives in CNS neurogenesis and repair. Pharmacol Ther 141: 21-31, 2014.

24. Zhao XH, Jin WL and Ju G: An in vitro study on the involvement of LINGO-1 and Rho GTPases in Nogo-A regulated differentiation of oligodendrocyte precursor cells. Mol Cell Neurosci 36 260-269, 2007.

25. Gard AL and Pfeiffer SE: Oligodendrocyte progenitors isolated directly from developing telencephalon at a specific phenotypic stage: Myelinogenic potential in a defined environment. Development 106: 119-132, 1989.
26. Haynes RL, Folkerth RD, Keefe RJ, Sung I, Swzeda LI, Rosenberg PA, Volpe JJ and Kinney HC: Nitrosative and oxidative injury to premyelinating oligodendrocytes in periventricular leukomalacia. J Neuropathol Exp Neurol 62: 441-450, 2003.

27. Jeffries MA, Urbanek K, Torres L, Wendell SG, Rubio ME and Fyffe-Maricich SL: ERK1/2 activation in preexisting oligodendrocytes of adult mice drives new myelin synthesis and enhanced CNS function. J Neurosci 36: 9186-9200, 2016.

28. Xiao J, Ferner AH, Wong AW, Denham M, Kilpatrick TJ and Murray SS: Extracellular signal-regulated kinase 1/2 signaling promotes oligodendrocyte myelination in vitro. J Neurochem 122: $1167-1180,2012$

29. Guardiola-Diaz HM, Ishii A and Bansal R: Erk1/2 MAPK and mTOR signaling sequentially regulates progression through distinct stages of oligodendrocyte differentiation. Glia 60: 476-486, 2012.

30. Gaesser JM and Fyffe-Maricich SL: Intracellular signaling pathway regulation of myelination and remyelination in the CNS. Exp Neurol 283: 501-511, 2016.

31. Ishii A, Furusho M, Dupree JL and Bansal R: Role of ERK1/2 MAPK signaling in the maintenance of myelin and axonal integrity in the adult CNS. J Neurosci 34: 16031-16045, 2014.

32. Fyffe-Maricich SL, Schott A, Karl M, Krasno J and Miller RH: Signaling through ERK1/2 controls myelin thickness during myelin repair in the adult central nervous system. J Neurosci 33 18402-18408, 2013.

33. Xie D, Shen F, He S, Chen M, Han Q, Fang M, Zeng H, Chen C and Deng Y: IL-1 $\beta$ induces hypomyelination in the periventricular white matter through inhibition of oligodendrocyte progenitor cell maturation via FYN/MEK/ERK signaling pathway in septic neonatal rats. Glia 64: 583-602, 2016.

34. Suzuki T, Hori N, Miyake T, Hori Y and Mochizuki K: Keratitis caused by a rare fungus, Malassezia restricta. Jpn J Ophthalmol 51: 292-294, 2007.

This work is licensed under a Creative Commons Attribution-NonCommercial-NoDerivatives 4.0 International (CC BY-NC-ND 4.0) License. 